\title{
Fabrication and Characterization of Polystyrene Surface with Atomic-Scale Surface Roughness*
}

\author{
Katsumi Shimizu, ${ }^{\dagger}$ Syoushi Higuchi, Amane Kitahara, Hikaru Terauchi, and Isao Takahashi ${ }^{\ddagger}$ \\ Department of Physics, School of Science and Technology, \\ Kwansei Gakuin University, 2-1 Gakuen, Sanda, Hyogo 669-1337, Japan \\ (Received 24 June 2012; Accepted 17 September 2012; Published 27 October 2012)
}

\begin{abstract}
A new approach for fabrication of polystyrene (PS) surface with atomic-scale surface roughness is presented. The obtained surface structure was investigated using X-ray reflectivity (XR) measurements in the temperature range between room temperature and $104^{\circ} \mathrm{C}$ which is close to the glass transition temperature $T_{\mathrm{g}}$ of bulk PS. At room temperature before heating, the specular XR profiles revealed a broad peak at $q_{z}=0.04 \AA^{-1}$. The XR at $q_{z}=0.04 \AA^{-1}$ increased with increasing temperature and showed an inflection point at ca. $65^{\circ} \mathrm{C}$. After cooling back to room temperature, thermal reversibility was evident in XR at $q_{z}=0.04 \AA^{-1}$. The thermally reversible XR observed in the present study would be peculiar to the surface of PS with infinite thickness.
\end{abstract}

[DOI: 10.1380/ejssnt.2012.591]

Keywords: X-ray scattering, diffraction, and reflection; Surface relaxation and reconstruction; Surface structure, morphology, roughness, and topography; Glass surfaces; Glass transition; Diffuse scattering

\section{INTRODUCTION}

Surface properties of polymeric materials are influenced by the molecular orientation and molecular dynamics in the surface regime at thicknesses up to a thousand angstroms. The characteristics in the surface regime are generally considered to be different from those in bulk regime; therefore, it is crucial to clarify the static structures and dynamic behavior of macromolecules in the topmost part of the surfaces having thickness ranging from nanometer-scale to micrometer-scale. Polystyrene (PS) is one of the most extensively investigated polymeric materials. Since the first observation of an anomaly in the thickness of ultrathin PS films below the bulk glass transition temperature $T_{\mathrm{g}}[1]$, many researchers have focused on the glass transition mechanism of PS thin films. Keddie et al. carried out ellipsometry measurements of supported PS thin films to investigate the thickness dependence of $T_{\mathrm{g}}$; they were the first to observe a depression in $T_{\mathrm{g}}$ with decreasing film thickness [2]. A similar trend of $T_{\mathrm{g}}$ depression in PS thin films has been observed using various techniques such as ellipsometry [3], X-ray reflectivity (XR) [4], positron lifetime measurements [5], and positron annihilation spectroscopy [6]. However, no significant thickness dependence of $T_{\mathrm{g}}$ depression was observed [7-9]; therefore, thickness dependence of $T_{\mathrm{g}}$ is still unclear. In surface/interface studies using supported thin films, interaction between thin films and substrates is always controversial. $T_{\mathrm{g}}$ reduction in free-standing $\mathrm{PS}$ thin films is considerably larger than that in supported films $[10,11]$ thus suggesting that $T_{\mathrm{g}}$ reduction depends on the configuration of macromolecules on substrates and the interaction with substrates. Brillouin light scattering studies revealed that the mechanical properties of free-standing PS thin films were consistent with those of the bulk [12]. Substrate effects on the physical properties of thin films were inves-

*This paper was presented at the 16th International Conference on Solid Films and Surfaces (ICSFS-16), University of Genoa, Genoa, Italy, 1-6 July, 2012.

†Corresponding author: shimizu@kwansei.ac.jp

$\ddagger$ Corresponding author: suikyo@kwansei.ac.jp tigated with different polymers and substrates [13-16]. Although XR measurements are one of the most effective tool to characterize the surface structures of polymeric materials, atomic-scale flatness of the sample surface is required; thus, thin films that are mainly formed by spin casting have been conventionally used. However, as indicated above, the experimental results using the supported thin films could be affected by characteristics such as the choice of substrate, the thickness of samples, and the solvents used in processing. Therefore, thin film structures on substrates might be different from the surface structures of bulk materials. In this report, we present the fabrication of the PS surface with atomic-scale surface roughness and the characterization of the surface using XR measurements.

\section{EXPERIMENTAL}

The polymers used in the present study are nearly monodispersed $\left(M_{\mathrm{w}} / M_{\mathrm{n}}=1.09\right)$ atactic PS with weightaveraged molecular weight $M_{\mathrm{w}}$ of $218,000 \mathrm{~g} / \mathrm{mol}$ (Polymer Source Inc.). For sample preparation, we employed a peeling-off technique for making a flat PS surface [17, 18]. An appropriate amount of PS was placed on $\mathrm{Si}(100)$ substrates and annealed in vacuum at $160^{\circ} \mathrm{C}$ for $6 \mathrm{~h}$. After the annealing, PS samples were quickly cool to $-20^{\circ} \mathrm{C}$ and were subsequently peeled off the substrates. The adherent side of the substrate was used as a sample surface and its dimensions were approximately $20 \times 20 \times 2 \mathrm{~mm}^{3}$. To characterize the PS surface, XR measurements were carried out using a high-resolution diffraction system with a stationary copper anode X-ray tube (Smart Lab system, Rigaku Co., Ltd., $40 \mathrm{kV} \times 30 \mathrm{~mA}$ ). The X-ray beam was monochromated using a multilayer mirror for taking a $\mathrm{CuK} \alpha$ radiation (wavelength $\lambda=1.5418 \AA$ ). The sample temperature was controlled by a custom-designed heater and a thyristor regulator temperature controller with a stability of $\pm 0.2^{\circ} \mathrm{C}$. The specular XR profiles were recorded by the longitudinal $q_{z}$ scan normal to the sample surface. Scattering vectors $q_{z}$ are defined as $q_{z}=$ $(4 \pi \sin \theta) / \lambda$, where $\theta$ and $\lambda$ are the incident angle and wavelength of the X-ray beam, respectively. The XR pro- 


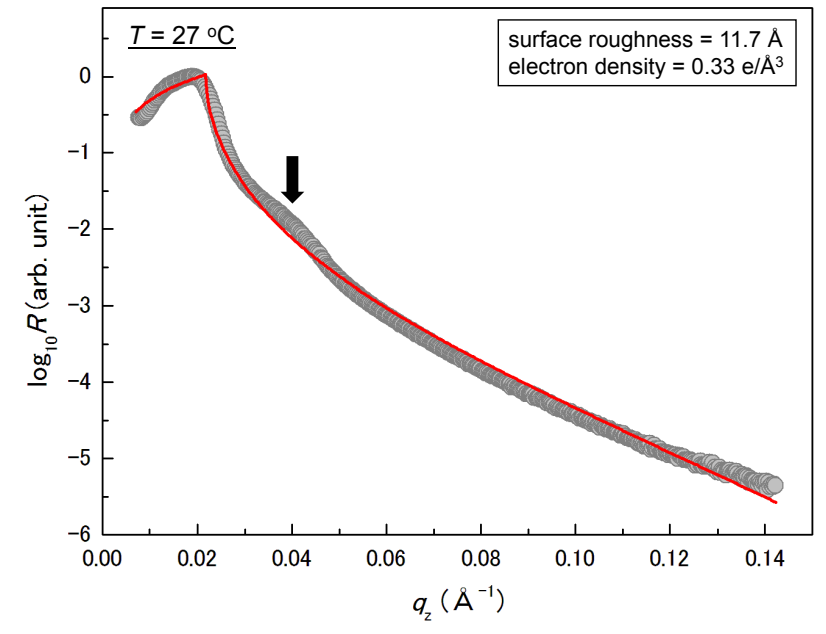

FIG. 1: Specular XR profile of the PS surface observed at room temperature $\left(27^{\circ} \mathrm{C}\right)$ before heating. The gray circles and the red line represent the observed XR and nonlinear least-squares regression, respectively. Momentum transfer $q_{z}$ is defined as $q_{z}=(4 \pi \sin \theta) / \lambda$, where $\theta$ and $\lambda$ are the incident angle and wavelength of the X-ray beam, respectively. The arrow indicates the position of the anomaly at $q_{z}=0.04 \AA^{-1}$.

files were recorded during the heating process and at room temperature $\left(27^{\circ} \mathrm{C}\right)$ before and after heating. The specular XR profiles were analyzed using a recurrence formula [19] with a two-layer (air and PS sample) model. The electron density and surface roughness of the PS samples were determined.

\section{RESULTS AND DISCUSSIONS}

Figure 1 shows the common logarithm of reflectivity $R$ of the PS surface as a function of scattering vectors $q_{z}$ at room temperature $\left(27^{\circ} \mathrm{C}\right)$ before heating. After nonlinear least-squares refinement, the electron density and surface roughness of the PS samples were converged to $0.33 e / \AA^{3}$ and $11.7 \AA$, respectively. The electron density of the PS sample is almost comparable to that of the PS thin films at room temperature [20] and the surface roughness is sufficiently small to evaluate the surface structure by XR measurements. Thus, the XR analysis revealed that the PS surface fabricated by the aforementioned method exhibits atomic-scale flatness. In the XR profile, a broad peak was reproducibly observed only at $q_{z}=0.04 \AA^{-1}$ (arrow in Fig. 1), whereas other peaks including a harmonic component of the broad peak could not be observed. To further examine the surface structures of PS, the XR profiles were observed at various elevated temperatures. Figure 2 shows the selected XR profiles of the PS surface at elevated temperatures and $27^{\circ} \mathrm{C}$ before and after heating. The broad peak at $q_{z}=0.04 \AA^{-1}$ shown in Fig. 1 became prominent with increasing temperature. The reflectivity in the higher $q_{z}$ regime increased with increasing temperature, which might be due to the smoothening of surface roughness at the elevated temperature. The inset in Fig. 2 shows the temperature dependence of reflectivity $\log _{10} R$ at $q_{z}=0.04 \AA^{-1}$. The reflectivity $\log _{10} R$ increased with increasing temperature

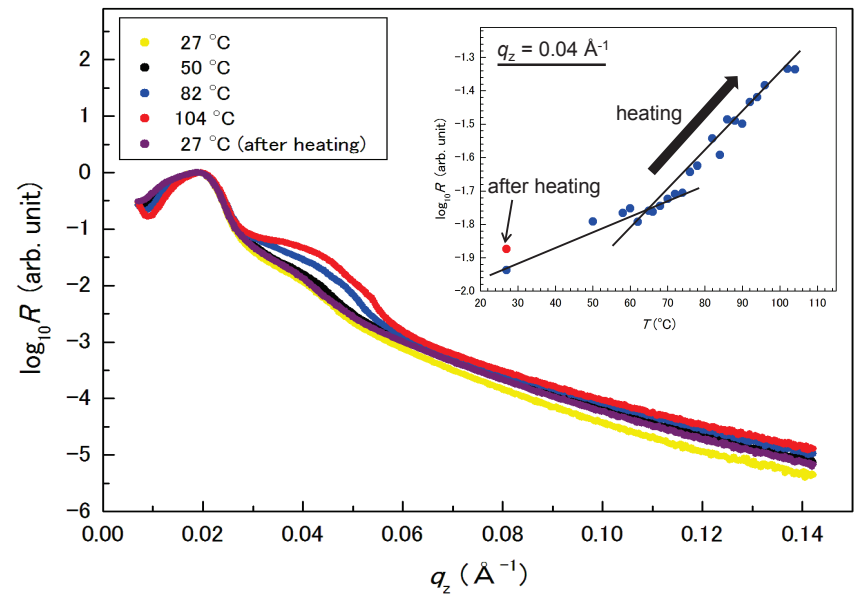

FIG. 2: Selected XR profiles of the PS surface at different temperatures: 27 (yellow), 50 (black), 82 (blue), 104 (red) observed in the heating process, and at $27^{\circ} \mathrm{C}$ after the heating (purple). Momentum transfer $q_{z}$ is defined as $q_{z}=(4 \pi \sin \theta) / \lambda$, where $\theta$ and $\lambda$ are the incident angle and wavelength of the X-ray beam, respectively. At $q_{z}=0.04 \AA^{-1}$, a broad peak is evident and becomes prominent with increasing temperature. The temperature dependence of common logarithm of reflectivity $R\left(\log _{10} R\right)$ at $q_{z}=0.04 \AA^{-1}$ is shown in the inset. The blue and red circles were recorded during the heating process and at room temperature after heating, respectively. The black straight lines are for visual guidance.

and showed an inflection point at ca. $65^{\circ} \mathrm{C}$. After cooling back to room temperature, the thermal reversibility was evident in the reflectivity $\log _{10} R$ at $q_{z}=0.04 \AA^{-1}$.

In the XR profiles, a thermally reversible broad peak was reproducibly observed at $q_{z}=0.04 \AA^{-1}$. A possible reason for its emergence is an undulated surface structure with heterogeneous long periodicity. The in-plane heterogeneous periodicity of surface undulation gives diffuse scattering normal to the $q_{z}$ direction in reciprocal space; therefore, the broad peak observed in the $q_{z}$ scan profiles might originate from the diffuse scattering component superimposed on the specular reflection along the $q_{z}$ direction. Another possibility of the emergence of the broad peak is the presence of a periodic layered structure normal to the PS surface; however, this is not probable because the periodic layered structure normal to the sample surface would exhibit a periodic interference fringe pattern in the XR profiles, as reported in previous studies $[1,4,7,20,23,24]$. We observed only one peak at $q_{z}=0.04 \AA^{-1}$ in the XR profiles. It is reported that the PS molecules in the vicinity of the sample surface are mobile compared to the molecules in the bulk [21]. The inflection shown in the inset of Fig. 2 might imply some changes in motion of PS molecules in the vicinity of the sample surface. The temperature at the inflection point $\left(\right.$ ca. $65^{\circ} \mathrm{C}$ ) is close in value to the lowest $T_{\mathrm{g}}$ of PS thin films $\left(57^{\circ} \mathrm{C}\right)[6]$. This might imply a relatively low $T_{\mathrm{g}}$ of the PS surfaces compared to the that of bulk [22] at ca. $100^{\circ} \mathrm{C}$. In the previous studies on supported PS thin films, the temperature dependence of the film thickness did not show a thermal reversibility in the range of the temperatures at which a conventional glass transition is observed. [1, 2, 20, 24]. The fictive temperature ob- 
served in the heating process seems to be different from $T_{\mathrm{g}}$ in supported PS thin films [20, 24]. The thermally reversible phenomena observed in this study (Fig. 2) would be peculiar to the surface regime of the bulk PS.

\section{CONCLUSIONS}

In conclusion, we present fabrication and characterization of PS surface with atomic-scale surface roughness. We observed the specular XR profiles of the obtained PS sample surface. The nonlinear least-squares refinement revealed the PS surface with atomic-scale surface flatness at room temperature. In the XR profile at room temperature, a broad peak was evident at $q_{z}=0.04 \AA^{-1}$. The peak became prominent with increasing temperature and showed a thermal reversibility after cooling back to room temperature. Further investigations are needed to clarify the origin of the thermally reversible surface structure inherent in PS.

\section{Acknowledgments}

This study was financially supported by The Promotion and Mutual Aid Corporation for Private Schools of Japan (The Science Research Promotion Fund in 2006-2008) and Kwansei Gakuin University (Kwansei Gakuin University Joint Research Fund, 2009).

[1] W. J. Orts, J. H. van Zanten, W. Wu, and S. K. Satija, Phys. Rev. Lett. 71, 867 (1993).

[2] J. L. Keddie, R. A. L. Jones, and R. A. Cory, Europhys. Lett. 27, 59 (1994)

[3] S. Kawana and R. A. L. Jones, Phys. Rev. E 63, 021501 (2001).

[4] T. Miyazaki, K. Nishida, and T. Kanaya, Phys. Rev. E 69, 061803 (2004).

[5] G. B. DeMaggio, W. E. Frieze, D. W. Gidley, M. Zhu, H. A. Hristov, and A. F. Yee, Phys. Rev. Lett. 78, 1524 (1997).

[6] Y. C. Jean, R. Zhang, H. Cao, J. Yuan, C. Huang, B. Nielsen, and P. Asoka-Kumar, Phys. Rev. B 56, R8459 (1997).

[7] M. Bhattacharya, M. K. Sanyal, Th. Geue, and U. Pietsch, Phys. Rev. E 71, 041801 (2005).

[8] L. Xie, G. B. DeMaggio, W. E. Frieze, J. DeVries, D. W. Gidley, H. A. Hristov, and A. F. Yee, Phys. Rev. Lett. 74, 4947 (1995).

[9] Y. Pu, H. White, M. H. Rafailovich, J. Sokolov, A. Patel, C. White, W. Wu, V. Zaitsev, and S. A. Schwarz, Macromolecules 34, 8518 (2001).

[10] J. A. Forrest, K. Dalnoki-Veress, J. R. Stevens, and J. R. Dutcher, Phys. Rev. Lett. 77, 2002 (1996).

[11] J. A. Forrest, K. Dalnoki-Veress, and J. R. Dutcher, Phys. Rev. E 56, 5705 (1997).
[12] J. A. Forrest, K. Dalnoki-Veress, and J. R. Dutcher, Phys. Rev. E 58, 6109 (1998).

[13] J. L. Keddie, R. A. L. Jones, and R. A. Cory, Faraday Discuss. 98, 219 (1994).

[14] Y. Zheng, D. Qi, N. Chandrasekhar, X. Gao, C. Troadec, and A. T. S. Wee, Langmuir 23, 8336 (2007).

[15] Y. Wang, M. Rafailovich, J. Sokolov, D. Gersappe, T. Araki, Y. Zou, A. D. L. Kilcoyne, H. Ade, G. Marom, and A. Lustiger, Phys. Rev. Lett. 96, 028303 (2006).

[16] J. H. van Zanten, W. E. Wallace, and W. Wu, Phys. Rev. E 53, R2053 (1996).

[17] M. Hegner, P. Wagner, and G. Semenza, Surf. Sci. 291, 39 (1993).

[18] C. Masens, J. Schulte, M. Phillips, and S. Dligatch, Microsc. Microanal. 6, 113 (2000).

[19] L. G. Parratt, Phys. Rev. 95, 359 (1954).

[20] C. Yang, A. Kitahara, and I. Takahashi, Polym. J. 41, 1036 (2009).

[21] W. E. Wallace, D. A. Fischer, K. Efimenko, W. Wu, and J. Genzer, Macromolecules 34, 5081 (2001).

[22] R. Greiner and F. R. Schwarzl, Rheol. Acta 23, 378 (1984).

[23] G. Reiter, Macromolecules 27, 3046 (1994).

[24] C. Yang and I. Takahashi, Polym. J. 43, 390 (2011). 